\title{
Effect of Glycemic Index and Glycemic Load on Atherosclerotic Stenosis and Stroke
}

\author{
Min Yuan ${ }^{1}$ and Huang-Yan Zhou ${ }^{2}$
}

Min Yuan and Huang-yan Zhou contributed equally to this work (Co-first author).

${ }^{1}$ Department of Neurology, Jiangxi provincial People's Hospital Affiliated to Nanchang University, Jiangxi, China

${ }^{2}$ Department of blood transfusion, Jiangxi Cancer Hospital, Jiangxi, China

Key words: Stroke, Atherosclerotic stenosis, Glycemic index, Glycemic load

\section{See article vol. 27: 1152-1159}

Recently, we read the article ${ }^{1)}$ published in the Journal of Atherosclerosis and Thrombosis by Peng and colleagues with great interest. The study observed increased 2.1-fold odds for higher carotid stenosis of glycemic index (GI) and an increased 1.8-fold risk of glycemic load $(\mathrm{GL})^{1)}$, but we want to present some questions about the above research.

During the last decade, there has been a well-recognized epidemiological link between high GL, GI, and the increased risk of stroke morbidity and mortality, while atherosclerotic stenosis is the main cause of stroke $^{2,3)}$. However, many studies have reported no significant association between high GI, GL, and stroke morbidity or mortality ${ }^{4,5)}$. Nonetheless, the evidence for this is limited. Although a number of clinical studies have assessed the association between GI, GL, and stroke outcome, the association between GI, $\mathrm{GL}$, and stroke is debated.

Cai et al. undertook a meta-analysis of prospective cohort studies and found that high GI was not associated with the risk of stroke events and that GL was a risk factor for strokes, but meta-regression suggested that no significant differences existed between gender and reference food ${ }^{6}$; whereas Rossi et al. showed that GL is merely an important determinant of the more common ischemic stroke, not of the hemorrhagic stroke ${ }^{7)}$. Levitan et al. ${ }^{4)}$ found that dietary GI and dietary GL were not associated with ischemic cardiovascular disease or mortality, but dietary GL was associated with a greater risk of hemorrhagic stroke.
Yu et al. conducted a prospective cohort study and suggested that high dietary GI and GL are associated with increased risks of total ischemic and hemorrhagic stroke in middle-aged and older urban Chinese women ${ }^{8)}$. Further, Song et al. ${ }^{9)}$ showed that high dietary GL was associated with the presence and burden of cerebral small vessel diseases in patients with acute cerebral infarction.

However, the recent study by Peng et al. ${ }^{1)}$ showed that there was no significant relationship between GI/ GL and cardiovascular disease risk factors using the Spearman test. We would like to point out some limitations and problems that are not mentioned in the article. First, the use of the food frequency questionnaire derived from the National Health and Nutrition Examination Surveys to evaluate patients' eating habits is highly subjective, and because the study is a cross-sectional study, the assessment of eating habits of patients who have been discharged from the hospital in the past will be considerably different. Second, for the baseline data included in the study, many of them have statistical differences, and there is no related propensity score matching in this paper. Third, there is no exclusion and explanation for patients who have previously taken statins, which is an important factor that affects hyperlipidemia and atherosclerotic stenosis. This makes us doubt the accuracy of the research results.

This recent controversial report challenges the association between GI and GL with atherosclerotic stenosis and stroke. It is therefore imperative to explore the attendant concerns. First, GI and GL have a substantially increased risk of atherosclerotic stenosis

Address for correspondence: Min Yuan, Department of Neurology, Jiangxi provincial People's Hospital Affiliated to Nanchang University, No. 152, Aiguo Road, Nanchang 330006, Jiangxi, China E-mail: yuanmin201314@sina.com.

Received: May 15, 2020 Accepted for publication: July 3, 2020

Copyright@2020 Japan Atherosclerosis Society

This article is distributed under the terms of the latest version of CC BY-NC-SA defined by the Creative Commons Attribution License. 
and stroke outcomes; future research can observe whether low-GL/GI diet daily could reduce the risk. As the diet patterns and dietary contributors to the GL vary in different populations that had an impact on the outcomes, other important factors should take into account in the future. Furthermore, a study-specific dose-response analysis based on the estimates presented for the different categories of carbohydrate intake, GI, and GL is needed.

In summary, future research should pay more attention to the exact pathogenetic mechanisms between GL/GI with atherosclerotic stenosis and stroke, and certain recommendations should be considered for the efficacy of the low-GL/GI diet daily for atherosclerotic stenosis and stroke outcomes in different sexes, countries, and ages. Large higher-quality prospective studies conducted with the use of standardized outcome measures in different levels are necessary to assess the association in the future.

\section{Acknowledgments}

The authors would like to thank all the people who participated in this study.

\section{Declaration of Conficting Interests}

The authors declared no potential conflicts of interest with respect to the research, authorship, and/ or publication of this article.

\section{Funding}

The present study was funded by the scientific research project of traditional Chinese medicine of Jiangxi Provincial Health and Family Planning Commission (NO. 2018A116). The authors thank the participants of this study for their important contributions.

\section{References}

1) Peng M, Li X, Liu Y, Zou M, Xia Y, Xu G. Dietary glycemic index and glycemic load in relation to atherosclerotic stenosis of carotid and cardiovascular risk factors in ischemic stroke patients. J Atheroscler Thromb, 2020; 27: 1152-1159

2) Sieri S, Brighenti F, Agnoli C, Grioni S, Masala G, Bendinelli B, Sacerdote C, Ricceri F, Tumino R, Giurdanella MC, Pala V, Berrino F, Mattiello A, Chiodini P, Panico S, Krogh V. Dietary glycemic load and glycemic index and risk of cerebrovascular disease in the EPICOR cohort. Plos One, 2013; 8: e62625

3) Hyvarinen M, Tuomilehto J, Mahonen M, Stehouwer CD, Pyorala K, Zethelius B, Qiao Q. Hyperglycemia and incidence of ischemic and hemorrhagic stroke-comparison between fasting and 2-hour glucose criteria. Stroke, 2009; 40: 1633-1637

4) Levitan EB, Mittleman MA, Hakansson N, Wolk A. Dietary glycemic index, dietary glycemic load, and cardiovascular disease in middle-aged and older Swedish men. Am J Clin Nutr, 2007; 85: 1521-1526

5) Oh K, Hu FB, Cho E, Rexrode KM, Stampfer MJ, Manson JE, Liu S, Willett WC. Carbohydrate intake, glycemic index, glycemic load, and dietary fiber in relation to risk of stroke in women. Am J Epidemiol, 2005; 161: 161169

6) Cai X, Wang C, Wang S, Cao G, Jin C, Yu J, Li X, Yan J, Wang F, Yu W, Ding F. Carbohydrate intake, glycemic index, glycemic load, and stroke: A meta-analysis of prospective cohort studies. Asia Pac J Public Health, 2015; 27: 486-496

7) Rossi M, Turati F, Lagiou P, Trichopoulos D, La Vecchia C, Trichopoulou A. Relation of dietary glycemic load with ischemic and hemorrhagic stroke: A cohort study in Greece and a meta-analysis. Eur J Nutr, 2015; 54: 215222

8) Yu D, Zhang X, Shu XO, Cai H, Li H, Ding D, Hong Z, Xiang YB, Gao YT, Zheng W, Yang G. Dietary glycemic index, glycemic load, and refined carbohydrates are associated with risk of stroke: A prospective cohort study in urban Chinese women. Am J Clin Nutr, 2016; 104: $1345-1351$

9) Song T, Chang Y, Kim A, Kim Y, Kim Y. High dietary glycemic load was associated with the presence and burden of cerebral small vessel diseases in acute ischemic stroke patients. Nutr Res, 2018; 51: 93-101 